Article

\title{
Gravimetric Vegetation Water Content Estimation for Corn Using L-Band Bi-Angular, Dual-Polarized Brightness Temperatures and Leaf Area Index
}

\section{Qi Wang ${ }^{1}$, Linna Chai ${ }^{1, *}$, Shaojie Zhao ${ }^{1}$ and Zhongjun Zhang ${ }^{2}$}

1 State Key Laboratory of Remote Sensing Science, Research Center for Remote Sensing and GIS, and School of Geography, Beijing Normal University, Beijing 100875, China;

E-Mails: wangqi7096@163.com (Q.W.); shaojie.zhao@bnu.edu.cn (S.Z.)

2 College of Information Science and Technology, Beijing Normal University, Beijing 100875, China; E-Mail: zzj@bnu.edu.cn

* Author to whom correspondence should be addressed; E-Mail: chai@bnu.edu.cn;

Tel.: +86-10-5880-7455-1687.

Academic Editors: Xin Li, Clement Atzberger and Prasad S. Thenkabail

Received: 9 March 2015 / Accepted: 7 August 2015 / Published: 17 August 2015

\begin{abstract}
In this study, an algorithm to retrieve the gravimetric vegetation water content (GVWC, \%) of corn was developed. First, the method for obtaining the optical depth from L-band (1.4 GHz) bi-angular, dual-polarized brightness temperatures (TB) for short vegetation was investigated. Then, the quantitative relationship between the corn optical depth, corn GVWC and corn leaf area index (LAI) was constructed. Finally, using the Polarimetric L-band Microwave Radiometer (PLMR) airborne data in the 2012 Heihe Watershed Allied Telemetry Experimental Research (HiWATER) project, the Global Land Surface Satellite (GLASS) LAI product, the height and areal density of the corn stalks, the corn GVWC was estimated (corn GLASS-GVWC). Both the in situ measured corn GVWC and the corn GVWC retrieved based on the in situ measured corn LAI (corn LAINET-GVWC) were used to validate the accuracy of the corn GLASS-GVWC. The results show that the GVWC retrieval method proposed in this study is feasible for monitoring the corn GVWC. However, the accuracy of the retrieval results is highly sensitive to the accuracy of the LAI input parameters.
\end{abstract}

Keywords: passive microwave; L-band; gravimetric vegetation water content (GVWC); optical depth; leaf area index (LAI); corn 


\section{Introduction}

Vegetation water content (VWC) is one of the most important parameters for evaluating the growth of vegetation. VWC has been widely used to monitor agricultural conditions, estimate biomass, etc. [1,2]. Moreover, VWC is a key parameter for retrieving soil moisture from active and passive microwave remote sensing data [3]. Therefore, developing a feasible VWC retrieval method is important for agricultural production, soil moisture monitoring, and biomass estimation.

Currently, many methods to retrieve VWC from visible- and infrared-band data are based on selecting bands that are the most sensitive to water content to construct vegetation moisture indices that can directly reflect the VWC. Statistical regression methods are used to establish empirical relationships between the VWC and these indices [4,5]. However, optical remote sensing data often contains only information for the vegetation canopy. Moreover, optical remote sensing methods are vulnerable to the effects of clouds and rain. Passive microwave remote sensing is an effective technique for monitoring land surface parameters because of its comprehensive, macro-scale, efficient, and real-time observations. This method can operate in all-weather conditions and during the entire day. The L-band (1.4 GHz) microwave can penetrate into the canopy layer and provide more vegetation structure information than the visible and infrared bands. This method has a significant advantage in terms of obtaining the VWC in densely vegetated regions [6].

In previous studies, Jackson and Schmugge [7] proposed a linear relationship between the VWC and the vegetation optical depth (Equation (1)).

$$
\tau=b \cdot w
$$

where $\tau$ is the vegetation optical depth, $w$ is the areal vegetation water content (unit: $\mathrm{kg} / \mathrm{m}^{2}$ ), and $b$ is a constant that depends on the vegetation type and observation frequency [8]. Microwave remote sensing technology has been widely applied in monitoring large-scale ground parameters. Therefore, the relationship between the VWC and vegetation optical depth in Equation (1) has been widely used to invert surface parameters in the microwave remote sensing method; this relationship plays an important role in the soil moisture and VWC inversions [9,10]. However, in previous studies, parameter $b$ was usually set according to empirical or prior knowledge [7,11]. Additionally, the VWC in Equation (1) is usually obtained using the optical remote sensing technique [7].

Generally, the VWC indicates the total water weight in vegetation per unit area (Unit: $\mathrm{kg} / \mathrm{m}^{2}$ ), whereas the GVWC indicates the gravimetric vegetation water content (Unit: \% or kg/kg) which can be calculated as the ratio of the difference between the fresh and dry weights of vegetation to the vegetation fresh weight $[12,13]$. The VWC is related to the total vegetation biomass above ground, whereas the GVWC can reflect the vegetation growing status.

Corn is one of the most important crops in China. The amount of corn produced in China is ranked second globally, and China is also the largest corn consumer. The planting area for corn is distributed widely in China. The corn water content must be monitored to evaluate the corn growth and to manage corn irrigation. In this study, an algorithm to retrieve the corn GVWC was developed. First, the method for obtaining the optical depth, which will be further used as an input parameter for corn GVWC retrieving, from L-band bi-angular, dual-polarized brightness temperatures (TB) for short vegetation was investigated. Then, the quantitative relationship between corn optical depth at L-band, corn GVWC and 
corn leaf area index (LAI) was constructed based on simulations. Finally, using the Polarimetric L-band Microwave Radiometer (PLMR) airborne data in the 2012 Heihe Watershed Allied Telemetry Experimental Research (HiWATER) project, the Global Land Surface Satellite (GLASS) LAI product, the height and areal density of the corn stalks, the corn GVWC was estimated (corn GLASS-GVWC). Both in situ measured corn GVWC and the corn GVWC retrieved based on in situ measured corn LAI (corn LAINET-GVWC) were used to validate the accuracy of the corn GLASS-GVWC.

The organization of this paper is as follows. In Section 2, the method to estimate the corn GVWC is introduced. This method is divided into two parts. The first part (Section 2.1) demonstrates the method to obtain the optical depth from L-band bi-angular, dual-polarized TB for short vegetation; whereas the second part (Section 2.2) investigates the quantitative relationship between the corn optical depth, corn GVWC and corn LAI. In Section 3, the study area, the remote sensing observations and the in situ measurements used in this study are introduced. Section 4 presents the results and discussions, followed by conclusions in Section 5 .

\section{Methodology}

Figure 1 shows the technique flow chart for the retrieval of the corn GVWC. The light-grey-colored panes show the optical depth retrieval procedure, and the dark-grey-colored panes show the development of a quantitative relationship between the corn GVWC, the corn optical depth at L-band, the corn LAI, and the height and areal density of the corn stalks. Then, by using PLMR TB, LAI data, measured height and areal density of the corn stalks and corn GVWC were retrieved. The difference between the yellow and cyan panels is the input LAI data. For yellow panels, corn GLASS-GVWC was retrieved using GLASS LAI. For cyan panels, corn LAINET-GVWC was retrieved using LAINET LAI.

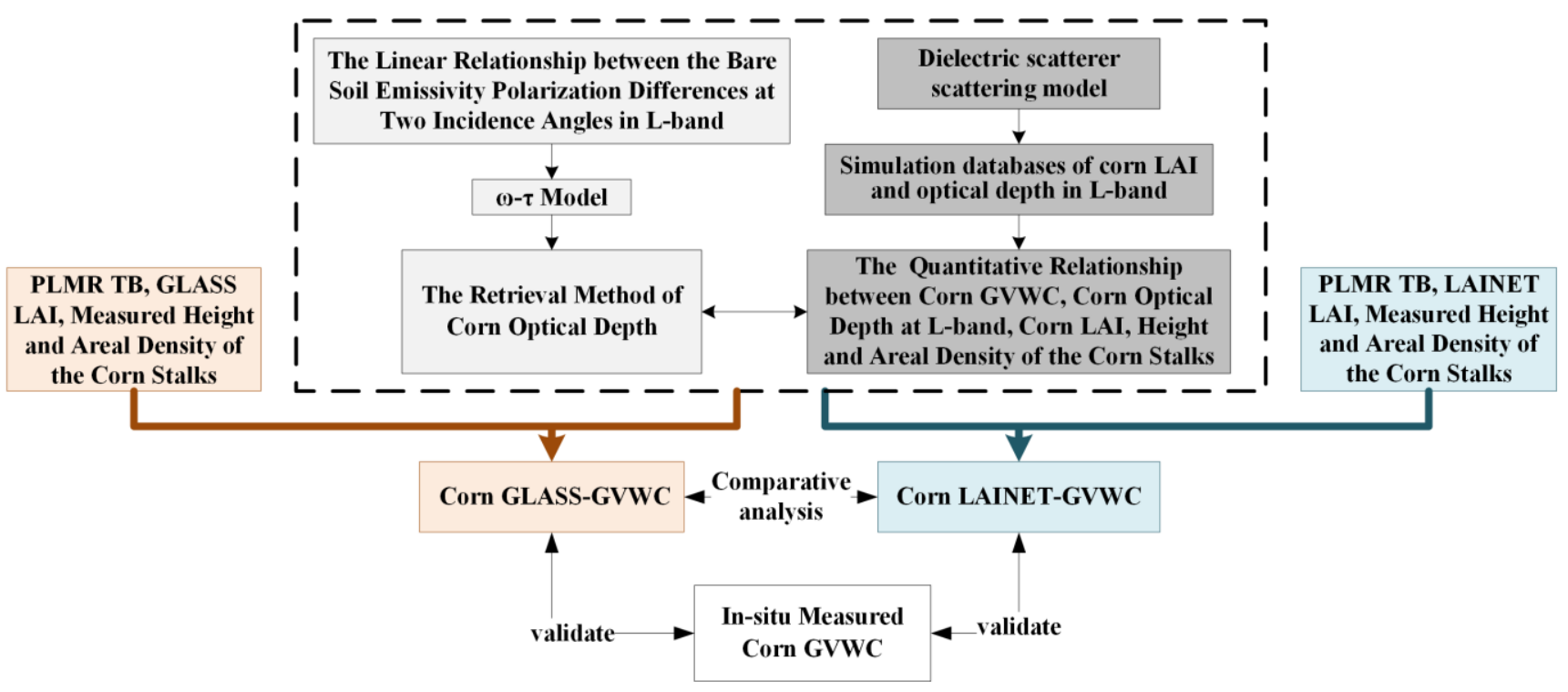

Figure 1. The technical flow chart for the retrieval of the corn GVWC. 


\subsection{The Optical Depth Retrieval Method for Short Vegetation}

In this study, we used the $\omega-\tau$ model to retrieve the vegetation optical depth. The $\omega-\tau$ model $[14,15]$ is the zero-order solution of the radiation transfer equation that neglects the scattering within the vegetation layer. This model is widely used to describe microwave radiation at low frequencies. When the vegetation coverage and atmospheric effects are not considered, the measured TB at a given viewing angle $(\theta)$ and frequency $(f)$ can be calculated using Equation (2):

$$
T b_{p}(f, \theta)=\varepsilon_{p}^{v}(f, \theta) \cdot T_{v}+\varepsilon_{p}^{v}(f, \theta) \cdot \Gamma_{p} \cdot\left(1-\varepsilon_{p}^{s}(f, \theta)\right) \cdot T_{v}+\varepsilon_{p}^{s}(f, \theta) \cdot \Gamma_{p} \cdot T_{s}
$$

where $\varepsilon$ is the emissivity; $\Gamma_{p}=\exp \left(-\tau_{p} / \cos \theta\right)$ is a one-way attenuation factor; $\theta$ and $\tau$ are the incidence angle and vegetation optical depth, respectively; and $f$ and $T b$ are the frequency (unit: GHz) and brightness temperature (unit: K), respectively. The superscripts $v$ and $s$ indicate the vegetation and soil components, respectively. The subscript $p$ refers to the polarization status $(\mathrm{V} / \mathrm{H})$. The emissivity of the vegetation canopy is given by

$$
\varepsilon_{p}^{v}=\left(1-\omega_{p}\right)\left(1-\Gamma_{p}\right)
$$

The parameters $T_{v}$ and $T_{s}$ are the vegetation and soil temperatures (unit: K), respectively. On the right side of Equation (2), the first term is the upward emission signal from the vegetation canopy, the second term is the downward vegetation emission signal reflected back by the soil surface after passing though the vegetation cover again, and the third term is the soil emission signal after it passes though the vegetation cover. Equation (2) can be expressed as a linear function of soil emissions (Equation (3)):

$$
T b_{p}(f, \theta)=\left[\varepsilon_{p}^{v}(f, \theta) \cdot\left(1+\Gamma_{p}\right) \cdot T_{v}\right]+\left[\Gamma_{p} \cdot\left(T_{s}-\varepsilon_{p}^{v}(f, \theta) \cdot T_{v}\right)\right] \cdot \varepsilon_{p}^{s}(f, \theta)
$$

At the passive microwave footprint scale, the temperatures of the short vegetation and soil can be considered equal, i.e., $T_{v}=T_{s}=T_{e}[16,17]$. Moreover, the observed vegetation canopy signals represent the overall effect of the mixture of the different vegetation canopy types present. When different types of vegetation canopies with different scatterer sizes, shapes and orientations are averaged, we may reasonably assume that no significant effects of the polarization dependence of the vegetation signals occur $[18,19]$. Furthermore, the single scattering albedo $(\omega)$ can be assumed to be 0 at L-band, as noted previously [20,21]. In summary, Equation (3) can be simplified to Equation (4):

$$
T b_{p}(\theta)=\left[\left(1-\Gamma^{2}(\theta)\right) \cdot T_{e}\right]+\left[\Gamma^{2}(\theta) \cdot T_{e}\right] \cdot \varepsilon_{p}^{s}(\theta)
$$

According to Equation (4), the polarization differences of the TB obtained at a specified incidence angle can be shown as the following:

$$
T b_{V}(\theta)-T b_{H}(\theta)=\Gamma^{2}(\theta) \cdot T_{e} \cdot\left(\varepsilon_{V}^{s}(\theta)-\varepsilon_{H}^{s}(\theta)\right)
$$

Then, the ratio of the polarization differences of the TB at $\theta_{1}$ and $\theta_{2}$ can be calculated via Equation (6):

$$
\frac{T b_{V}\left(\theta_{2}\right)-T b_{H}\left(\theta_{2}\right)}{T b_{V}\left(\theta_{1}\right)-T b_{H}\left(\theta_{1}\right)}=\frac{e^{-2 \tau / \cos \theta_{2}}}{e^{-2 \tau / \cos \theta_{1}}} \cdot \frac{\varepsilon_{V}^{s}\left(\theta_{2}\right)-\varepsilon_{H}^{s}\left(\theta_{2}\right)}{\varepsilon_{V}^{s}\left(\theta_{1}\right)-\varepsilon_{H}^{s}\left(\theta_{1}\right)}
$$

To characterize the relationships between the bare soil emissivity polarization differences at two incidence angles at L-band, a simulated surface emission database at $1.4 \mathrm{GHz}$ was generated using the Advanced Integrated Emission Model (AIEM) [22]. The simulated bare soil emissivity includes a wide 
range of soil parameters (Table 1). When analyzing the simulations, good relationships were found between the emissivity polarization differences at two incidence angles (Equation (7)) when the difference between the two incidence angles is small (Figure 2).

$$
\varepsilon_{V}^{s}\left(\theta_{2}\right)-\varepsilon_{H}^{s}\left(\theta_{2}\right)=\beta\left(\theta_{1}, \theta_{2}\right) \cdot\left[\varepsilon_{V}^{s}\left(\theta_{1}\right)-\varepsilon_{H}^{s}\left(\theta_{1}\right)\right]
$$

where $\varepsilon_{p}^{S}$ is the soil emissivity under $p$ polarization $(\mathrm{H} / \mathrm{V}) ; \theta_{1}$ and $\theta_{2}$ are the two incidence angles; and $\beta\left(\theta_{1}, \theta_{2}\right)$ is a coefficient that can be obtained via the linear regression of the bare soil emissivity polarization differences at two incidence angles.

Table 1. Parameter ranges in the simulation database for bare soil emissivity.

\begin{tabular}{ccccc}
\hline Parameters & Unit & Min & Max & Step \\
\hline Incident angles & $\circ$ & 1 & 60 & 1 \\
Soil moisture content & $\%$ & 2 & 44 & 2 \\
RMs heights & $\mathrm{cm}$ & 0.25 & 3 & 0.25 \\
Correlation lengths & $\mathrm{cm}$ & 2.5 & 30 & 2.5 \\
\hline
\end{tabular}
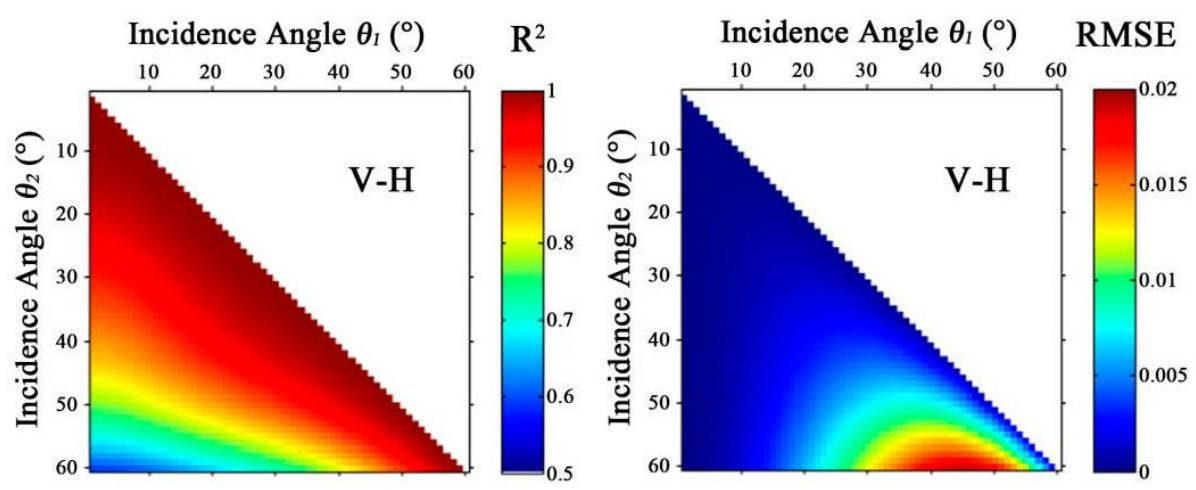

Figure 2. The determination coefficient $\left(\mathrm{R}^{2}\right)$ and root-mean-square error (RMSE) between the simulated bare soil emissivity polarization differences at any two incidence angles.

By combining Equations (6) and (7), Equation (8) can be derived. This equation can be used to calculate the L-band optical depth for short vegetation based on L-band bi-angular passive microwave TB.

$$
\tau=\frac{1}{2} \cdot \ln \left[\beta\left(\theta_{1}, \theta_{2}\right) \cdot \frac{T b_{V}\left(\theta_{1}\right)-T b_{H}\left(\theta_{1}\right)}{T b_{V}\left(\theta_{2}\right)-T b_{H}\left(\theta_{2}\right)}\right] \cdot \frac{\cos \theta_{1} \cdot \cos \theta_{2}}{\cos \theta_{1}-\cos \theta_{2}}
$$

where $\tau$ is the optical depth of the short vegetation; $\theta_{1}$ and $\theta_{2}$ are two incidence angles; $T b_{p}$ is the brightness temperature (unit: $K$ ); and the subscript $p$ indicates the polarization status (H/V). It should be noted that, Equation (8) is only applicable to short vegetation because only under this circumstance, it is reasonable to assume the single scattering to be zero.

\subsection{A Quantitative Relationship between the Corn GVWC, the Corn Optical Depth at L-band, the} Corn LAI, and the Height and Areal Density of the Corn Stalks

To study the quantitative relationship between the corn GVWC, the corn optical depth at L-band, the corn LAI, and the height and areal density of corn stalks, a simulated optical depth and LAI database for corn was generated. The corn leaves were approximated to dielectric circular disks by ensuring the 
surface areas of corn leaves were equal to the total area of the corresponding circular disks [23,24]. In other words, the long and curving shape of corn leaves can be considered as several disks with randomly distributed orientations with the identical area. The corn stalks were approximated to vertically distributed dielectric circular cylinders [25]. The database covers a wide range of corn parameters during various growth stages (Table 2). These parameters were set according to in situ measurements obtained from the seedling to the mature stage during the HiWATER project.

Table 2. The configuration of corn parameters for generating the database.

\begin{tabular}{cccccc}
\hline & Parameters & Unit & Min & Max & Step \\
\hline \multirow{4}{*}{ Corn Leaves } & Areal density $\left(M_{d}\right)$ & $\mathrm{m}^{-2}$ & 50 & 1250 & 150 \\
& Radius $\left(r_{d}\right)$ & $\mathrm{m}$ & 0.005 & 0.065 & 0.01 \\
& Thickness $\left(h_{d}\right)$ & $\mathrm{m}$ & 0.0001 & 0.0004 & 0.0001 \\
& GVWC $\left(w_{d}\right)$ & $\%$ & 60 & 90 & 5 \\
\hline \multirow{5}{*}{ Corn Stalks } & Areal density $\left(M_{c}\right)$ & $\mathrm{m}^{-2}$ & 5 & 9 & 1 \\
& Radius $\left(r_{c}\right)$ & $\mathrm{m}$ & 0.01 & 0.025 & 0.005 \\
& Length $\left(h_{c}\right)$ & $\mathrm{m}$ & 0.1 & 2 & 0.1 \\
& GVWC $\left(w_{c}\right)$ & $\%$ & 60 & 90 & 5 \\
\hline
\end{tabular}

Based on Table 2, we used the Mätzler vegetation dielectric model [26] to simulate the permittivities of corn scatterers. Additionally, we used the Generalized Rayleigh-Gans approximation (GRG) [27,28] to obtain the simulated optical depth of the randomly and uniformly distributed circular disks and the Infinite Length Cylinder approximation (IL) $[25,28]$ to obtain the simulated optical depth of the vertically distributed circular cylinders. We then established a simulated LAI database for the corn leaves based on the surface area of the leaves, and we established a simulated LAI database for the corn stalks based on the cross-sectional area of the stalks. Finally, we combined the simulated optical depths of the corn leaves and stalks with the identical GVWC $\left(w_{c}=w_{d}\right)$, as well as the simulated LAI. Thus, we generated the simulated optical depth and LAI of the entire corn.

By analyzing the simulated optical depth and LAI of the corn, the corn optical depth $(\tau)$ can be calculated as follows (Equation (9)).

$$
\tau=\left(a^{\prime} \cdot L A I+c^{\prime}\right) \cdot w+b^{\prime} \cdot L A I+d^{\prime}
$$

where $\tau$ is the corn optical depth, $w$ is the corn GVWC (unit: \%), LAI is the corn leaf area index, $a^{\prime}=0.1091, b^{\prime}=-0.027$, and $c^{\prime}$ and $d^{\prime}$ depend on the height $\left(h_{c}\right.$, unit: $\left.\mathrm{m}\right)$ and areal density of the corn stalks $\left(M_{c}\right.$, unit: $\left.\mathrm{m}^{-2}\right)$ (Equations (10) and (11)).

$$
\begin{aligned}
c^{\prime} & =\left(c_{11} \cdot h_{c}+c_{12}\right) \cdot M_{c}+c_{2} \\
d^{\prime} & =\left(d_{11} \cdot h_{c}+d_{12}\right) \cdot M_{c}+d_{2}
\end{aligned}
$$

where $c_{11}=-0.0363, c_{12}=0.0011, c_{2}=-0.0406, d_{11}=0.0737, d_{12}=-0.002$, and $d_{2}=0.0178$.

A comparison between the corn optical depths simulated by the model and calculated by Equation (9) (Figure 3) indicated that Equation (9) displays an acceptable accuracy when calculating the corn optical depth with a $\mathrm{R}^{2}$ of 0.9947 and a RMSE of 0.0208 . 


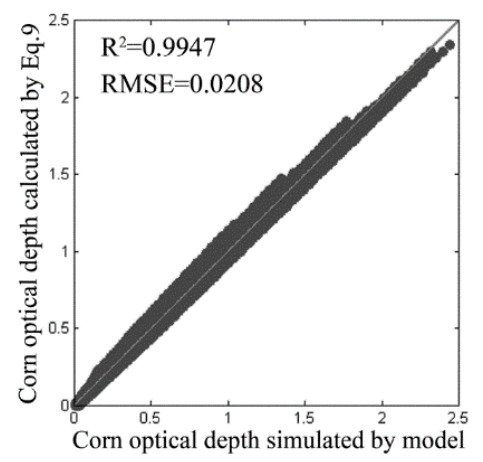

Figure 3. The correlation between the corn optical depth simulated by the model and that calculated by Equation (9).

\section{Study Region and Datasets}

\subsection{Description of the Study Region}

The Heihe River Basin, located in the middle of the Hexi Corridor $\left(98^{\circ}-101^{\circ} 30^{\prime} \mathrm{E}, 38^{\circ}-42^{\circ} \mathrm{N}\right)$, is the second largest inland river basin in Northwest China. Spanning three types of natural environments (the Qilian geosynclinal fold belt, the Alashan anteclise and the block belt of Beishan, the depression basin of the Hexi Corridor), the basin is approximately $821 \mathrm{~km}$ long with an area of $142,900 \mathrm{~km}^{2}$. The HiWATER project was conducted in 2012 in the Heihe River Basin to improve the observations of hydrological and ecological processes, to build a world-class watershed observation system, and to enhance the applicability of remote sensing in integrated eco-hydrological studies and basin-scale water resource management [29,30].

The study area is located in the middle reaches of the Heihe River Basin in Zhangye $\left(100^{\circ} 9^{\prime} 0.31^{\prime \prime}-100^{\circ} 36^{\prime} 4.74^{\prime \prime} \mathrm{E}, 38^{\circ} 43^{\prime} 22.25^{\prime \prime}-39^{\circ} 9^{\prime} 19.11^{\prime \prime} \mathrm{N}\right)$. The $1960 \mathrm{~km}^{2}$ study area has gently sloping terrain. The 2012 land cover data for the Heihe River Basin [31], with a spatial resolution of $30 \mathrm{~m}$, were used in this study. The predominant land use types in the study region were croplands and urban areas. The primary crop was corn, and spring wheat and barley were planted in small areas (Figure 4).

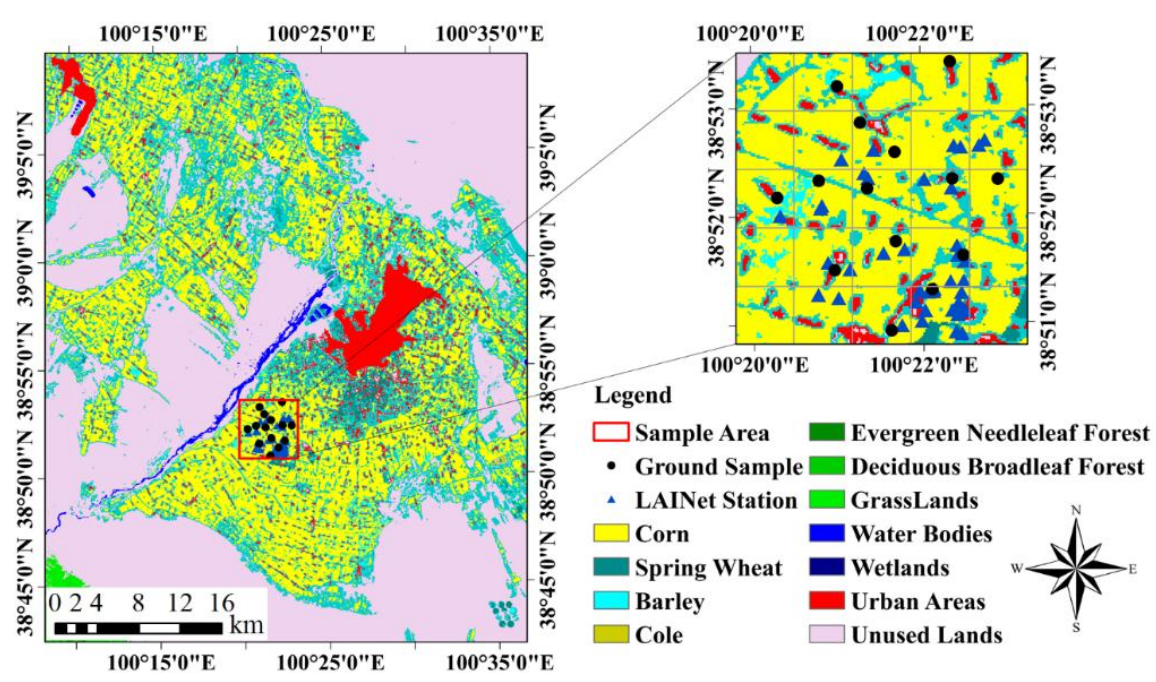

Figure 4. The entire study region, the validation region, the LAINet wireless network stations, and the ground sample points of the corn GVWC. 


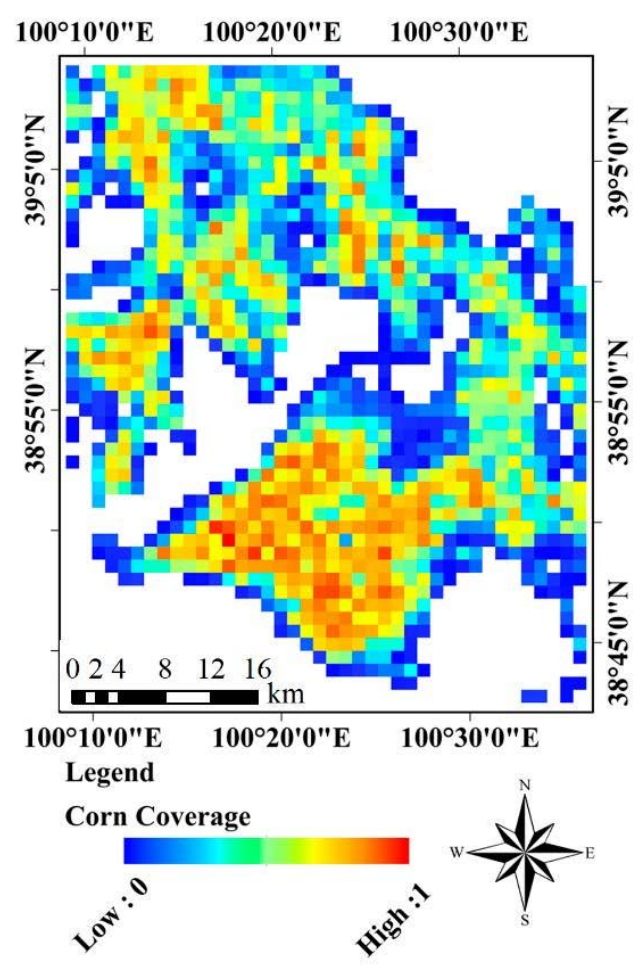

a

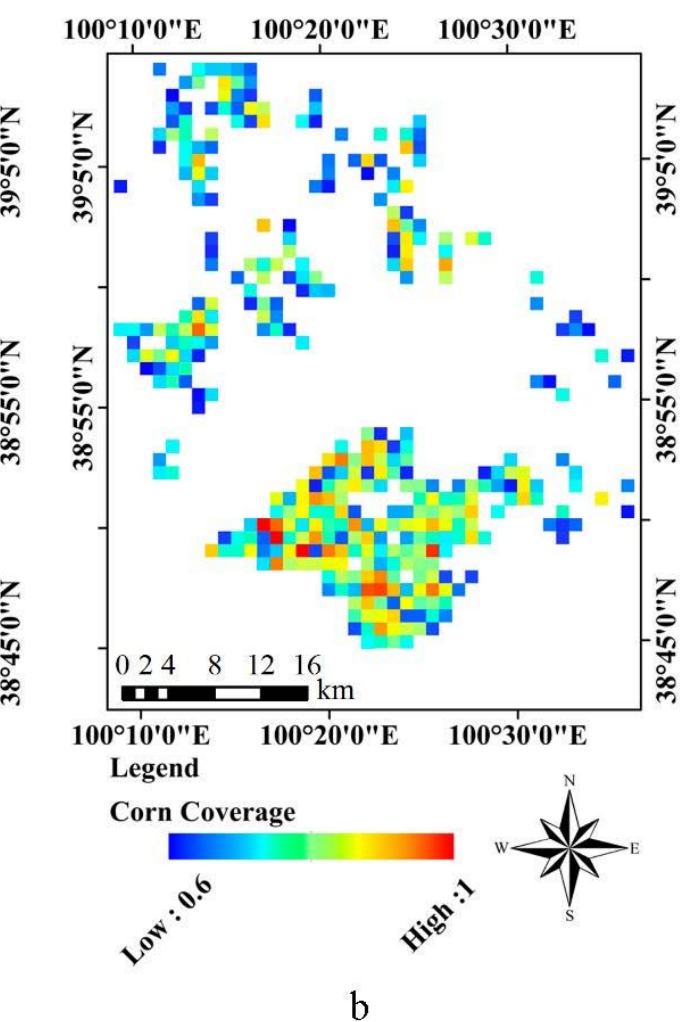

Figure 5. The corn coverage within the study region ((a) 1-km spatial resolution) and the pixels within the study region for which the corn coverage was greater than $60 \%((\mathbf{b}) 1-\mathrm{km}$ spatial resolution).

We calculated the corn coverage of each 1-km pixel in the study region based on the 2012 land cover data for the Heihe River Basin [31]. The results are shown in Figure 5a using the UTM WGS84 projection. To minimize the heterogeneity in the land cover and to improve the retrieval accuracy of the corn GVWC, we selected the pixels for which the corn coverage was greater than $60 \%$ (Figure $5 \mathrm{~b}$ ) and retrieved the corn GVWC within these pixels.

\subsection{Datasets}

\subsubsection{Remote Sensing Observations}

The HiWATER passive microwave aircraft program provided L-band multi-angle land surface observations using a PLMR (provided by Monash University, Australia) instrument [32,33]. The PLMR has a channel at $1.4 \mathrm{GHz}$ with a dual polarization $(\mathrm{V} / \mathrm{H})$. The PLMR has six incidence angles, i.e., $\pm 7.5^{\circ}$, $\pm 22^{\circ}$, and $\pm 38^{\circ}$. The accuracy of the PLMR was estimated to be higher than 2 and $3 \mathrm{~K}$ in the $\mathrm{H}$ and $\mathrm{V}$ polarizations, respectively [34]. The spatial resolution of the flight data is approximately $1 \mathrm{~km}$ for a flight altitude of $3 \mathrm{~km}$. The radiometer was calibrated before and after each flight. Nine flights were conducted as a part of the HiWATER project between 30 June 2012 and 2 August 2012. The flight area was a subcatchment of the Heihe River Basin. We extracted the PLMR TB at incidence angles of $\pm 7.5^{\circ}, \pm 22^{\circ}$, and $\pm 38^{\circ}$ within the study region during data processing without considering the effect of the observed azimuth on the brightness temperature observations; therefore, no observed azimuth differences were noted between the PLMR observations for the identical incidence angles on the left and right sides. After 
processing, the incidence angles of the TB were $7.5^{\circ}, 22^{\circ}$, and $38^{\circ}$. We performed quality control for the TB obtained by PLMR based on the actual conditions of the experiment, and we selected the TB in the range of 150 to $350 \mathrm{~K}$. The UTM WGS-84 projection was used for the PLMR TB after eliminating noise, and the spatial resolution was aggregated to $1 \mathrm{~km}$. The PLMR TB after aggregation and the corn coverage data (Figure 5a) in Section 3.1 were spatially matched. Considering the overlap between the flights and the study region, the flights on 30 June, 3 July, 4 July, 7 July, 10 July, 26 July, 1 August, and 2 August 2012 were used in this study [35]. However, the TB at the $7.5^{\circ}$ incidence angle may be affected by the emission form the plane on which the PLMR is carried. Therefore, the TB at the incidence angles of $22^{\circ}$ and $38^{\circ}$ were used to retrieve the corn GVWC in this study. According to Equation (7) and Figure 2 in Section 2.1, the coefficient $\beta(38,22)$ is 0.3014 , with a $\mathrm{R}^{2}$ of 0.9632 and a RMSE of 0.0024 .

The GLASS LAI product [36] provided by the Center for Global Change Data Processing and Analysis of Beijing Normal University was used in this study. The 8-day, 1-km resolution GLASS LAI product used the sinusoidal projection. The GLASS LAI product was retrieved using the General Regression Neural Networks (GRNN), which was trained by the fused time series LAI values from the Moderate Resolution Imaging Spectroradiometer (MODIS) and Carbon Cycle and Change in Land Observational Products from an Ensemble of Satellites (CYCLOPES) LAI products. The GLASS LAI product is spatially complete and temporally continuous [37]. We converted the projection of the GLASS LAI product from the sinusoidal projection to the UTM WGS84 projection using the nearest-neighbor resampling method. Consequently, the LAI product, the corn coverage data (Figure 5a) in Section 3.1 and the PLMR TB after aggregation were spatially matched.

\subsubsection{In-Situ Measurements}

A ground validation region [38] of approximately $5 \times 5 \mathrm{~km}^{2}$ was established in the PLMR flight region (Figure 4). Corn was the predominant land cover type in the validation region. Fifteen ground sampling points were deployed for the corn GVWC in this area. The distribution of the ground sampling points is shown in Figure 4. The corn samples at each ground sampling point were obtained every seven days, and the oven drying method was used to measure the corn GVWC. This GVWC value will be further used to validate the accuracy of the retrieved corn GVWC. In total, 15 sets of measurements from 14 May 2012 to 28 August 2012 were obtained. The measured corn GVWC spanned the corn growth process from the seedling to the mature stage [39].

To further analyze the corn GLASS-GVWC, the GLASS LAI data were replaced with the in situ measured corn LAI data [40] to retrieve the other set of corn GVWC, i.e., the corn LAINET-GVWC, using Equation (9). The LAI data were obtained from the LAINet wireless network [41] distributed within the validation region. The LAINet contains 42 stations. The distribution of the LAINet stations is shown in Figure 4. The LAINet observations were conducted between 25 June 2012 and 24 August 2012 (61 days).

Additionally, the program of observing the biomass in the middle reaches of the Heihe River Basin during the HiWATER project provided the density and height of the corn stalks within the validation region. The measured corn heights covered the growth process of the corn from the seedling to the mature stage, and the density of the corn stalks was 15 plants $/ \mathrm{m}^{2}$. Therefore, these measurements provide a statistical average height for the corn for specific dates within the study region. The planting time of 
the corn within the study region was late April 2012 (day of year (doy): approximately 115). The corn was in the seedling stage [42] from sowing to late May (doy: approximately 145). At this stage, the corn heights increased slowly. The corn was in the elongating stage [42] from late May (doy: approximately 145) to mid-July (doy: approximately 190). In this stage, the corn heights increased rapidly. The corn heights reached their maximum value of approximately $180 \mathrm{~cm}$ in late July (doy: approximately 195). At the end of the lifecycle of the corn, the corn begins to wither. The corn height decreased slightly from late July (doy: approximately 200) to late September (doy: approximately 270). To quantify the correlation of the corn height and time within the study region in 2012, the relationship between the measured corn height and time was fitted by a piecewise function (Figure 6). According to the measured data and observation records during the HiWATER project, we set doy $=195$ (the maximum corn heights occurred on doy $=195$ ) as the turning point of the piecewise function. The piecewise function was used to fit the measured corn heights. We used a quadratic function to fit the measured corn heights when doy $\leq 195$ (Equation $(12)$ ), $\mathrm{R}^{2}=0.996$, and $\mathrm{RMSE}=0.0342$ ) and a linear function to fit the measured corn heights when doy $>195$ (Equation $(13), \mathrm{R}^{2}=0.9972$, and RMSE $\left.=0.0205\right)$.

$$
\begin{gathered}
h_{c}=0.000459388 \cdot \operatorname{doy}^{2}-0.12215 \cdot \operatorname{doy}+8.19517(\text { doy } \leq 195) \\
h_{c}=-0.0012 \cdot \operatorname{doy}+2.0237(\text { doy }>195)
\end{gathered}
$$

where $h_{c}$ indicates the corn height (unit: $\mathrm{m}$ ), and doy indicates the day of year.

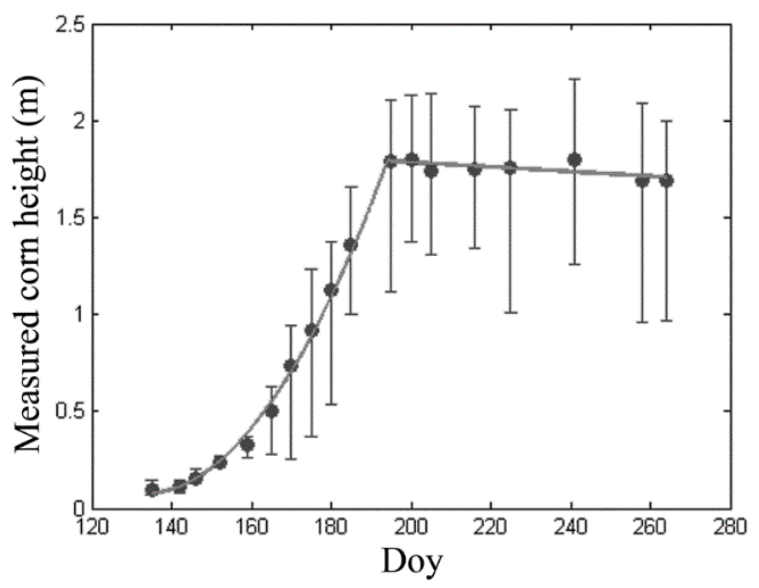

Figure 6. Corn height changes over time in the study region in 2012.

\section{Results and Discussions}

\subsection{The Retrieval of Corn GLASS-GVWC}

Based on the method described in Section 2, the corn GLASS-GVWC were retrieved using the PLMR TB, the GLASS LAI product, and the height and areal density of the corn stalks within the pixels with a corn coverage greater than $60 \%$ (Figure 7) in the study region on the eight flight days. Because of the different PLMR flight plans during HiWATER, each flight covers a different area within the study region. Therefore, the spatial distribution of corn GLASS-GVWC in Figure 7 shows a substantial day-to-day difference. 

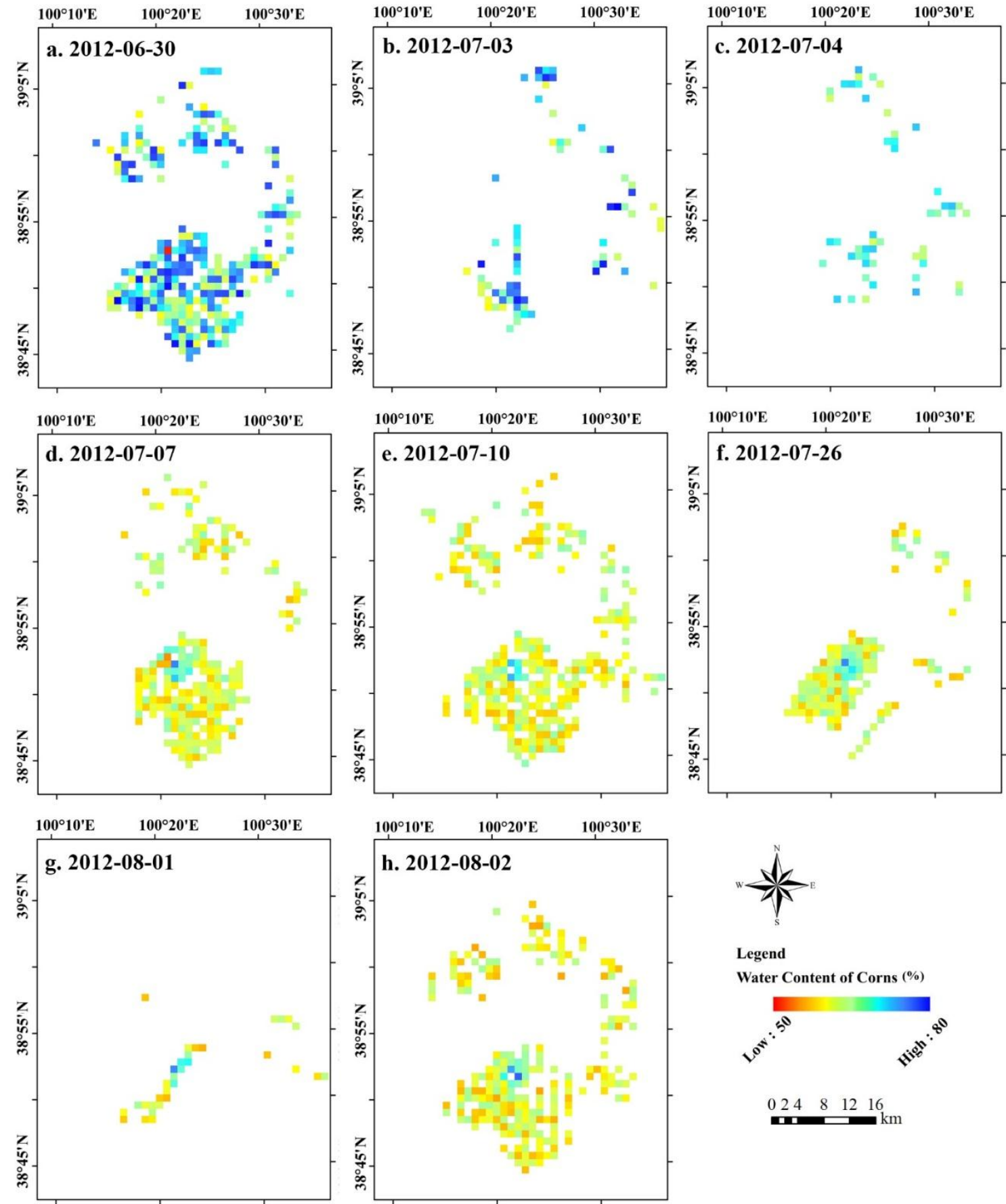

Figure 7. The corn GLASS-GVWC (unit: \%) in the study region on the day of flights.
(a) 2012-06-30;
(b) 2012-07-03;
(c) 2012-07-04;
(d) 2012-07-07;
(e) 2012-07-10;
(f) 2012-07-26; (g) 2012-08-01; (h)
(h) 2012-06-30; (a) 2012-08-02.

In the validation region, the corn GLASS-GVWC of each pixel was validated by coincident measurements of the corresponding ground sampling points on the day of the PLMR flights. Figure 8 shows that, in the validation region, the corn GLASS-GVWC were statistically lower than the ground corn GVWC measurements. We will discuss the reason for this bias in detail in the next section. Furthermore, we respectively averaged all of the corn GLASS-GVWC results and the measured corn GVWC for each day (Figure 9). The daily averaged corn GLASS-GVWC is lower than the measured corn GVWC. However, the time-variation trends were similar, i.e., either the GLASS-GWVC or the 
measured GVWC decreased gradually during the growth of the corn. This result is mainly caused by the increasing percentage of organics and dry matter within the corn body during corn growth. We calculated the deviations between the measured corn GVWC and the corn GLASS-GVWC. The results show that the $\mathrm{R}^{2}$ is 0.306 , and the RMSE is $5.61 \%$.

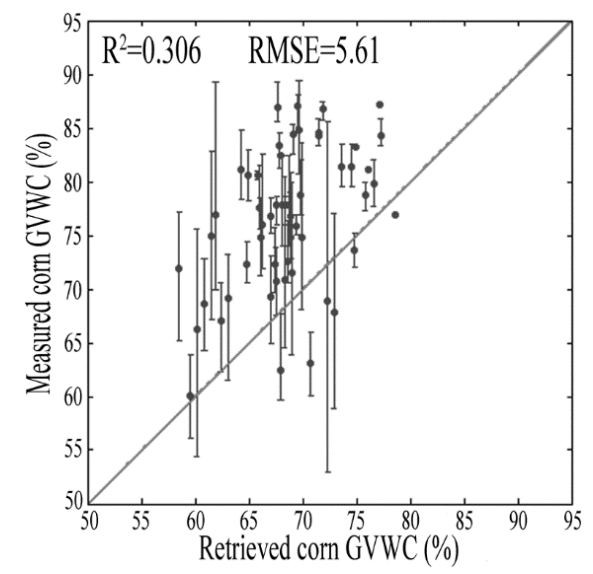

Figure 8. A comparison between the corn GLASS-GVWC and the corn GVWC measured by the ground sampling points in the study region.

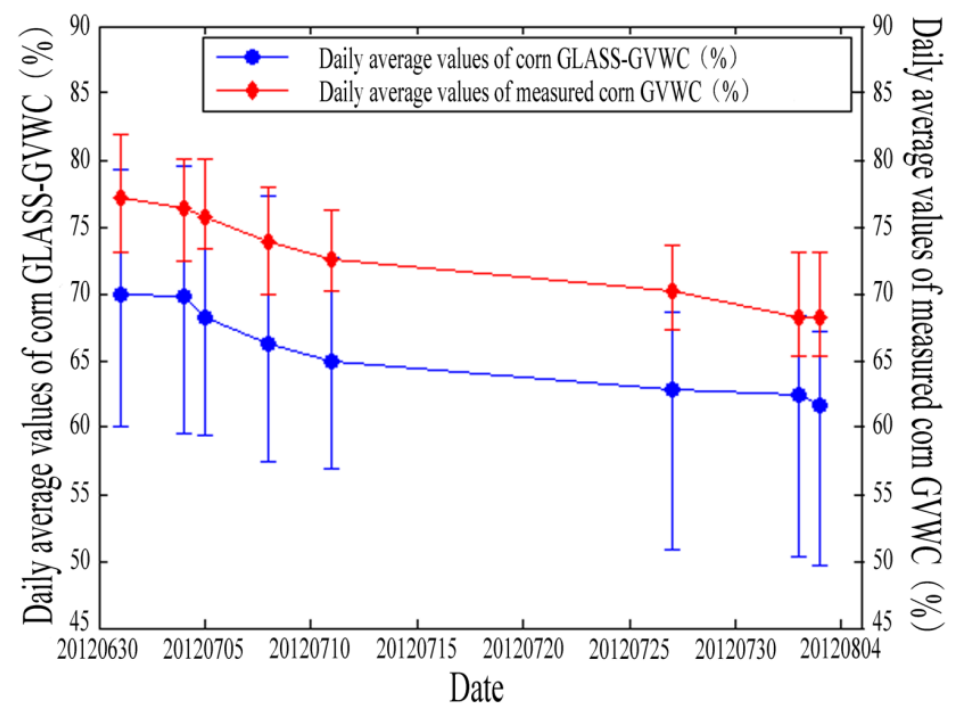

Figure 9. Daily average of the corn GLASS-GVWC and measured corn GVWC.

\subsection{Discussion}

To further validate the corn GVWC retrieval method, we retrieved another set of corn GVWC (corn LAINET-GVWC) by replacing the GLASS LAI with LAINet observation data within the validation region. The corn LAINET-GVWC was validated using the coincident measurements of the corresponding ground sample points on the day of the PLMR flights. Comparisons between the ground-measured corn GVWC and the corn LAINET-GVWC (Figure 10) shows that the measured corn GVWC and the corn LAINET-GVWC were uniformly distributed along the 1:1 line. This result indicates that the corn LAINET-GVWC agrees well with the measured corn GVWC with a $\mathrm{R}^{2}$ of 0.7768 and a RMSE of $3.13 \%$. 


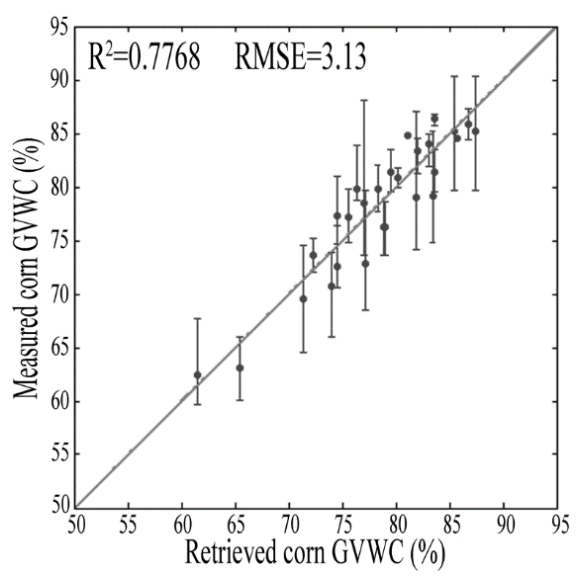

Figure 10. The comparison between the corn LAINET-GVWC and the corn GVWC measured by the ground sampling points.

Therefore, it is logical to conjecture that the differences between the corn GLASS-GVWC and LAINET-GVWC at the same time and place can be explained by the different input LAI data source in the two retrieval processes. We compared the GLASS LAI product with the corresponding LAINet observation data in the validation region. The results show that the GLASS LAI was statistically lower than the LAINet observation data (Figure 11a).

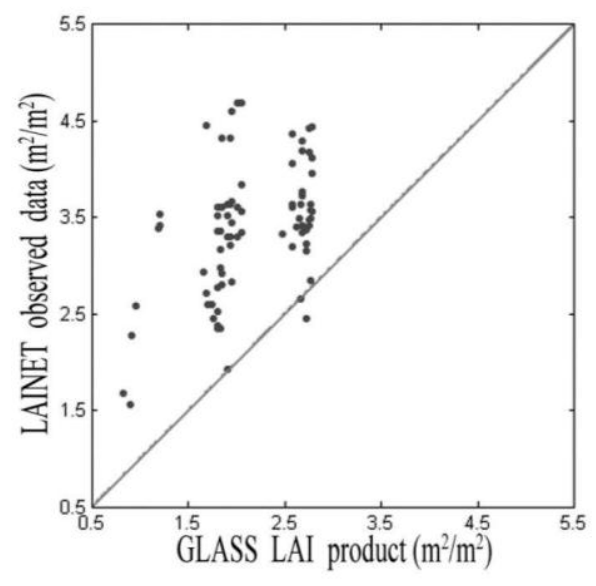

a

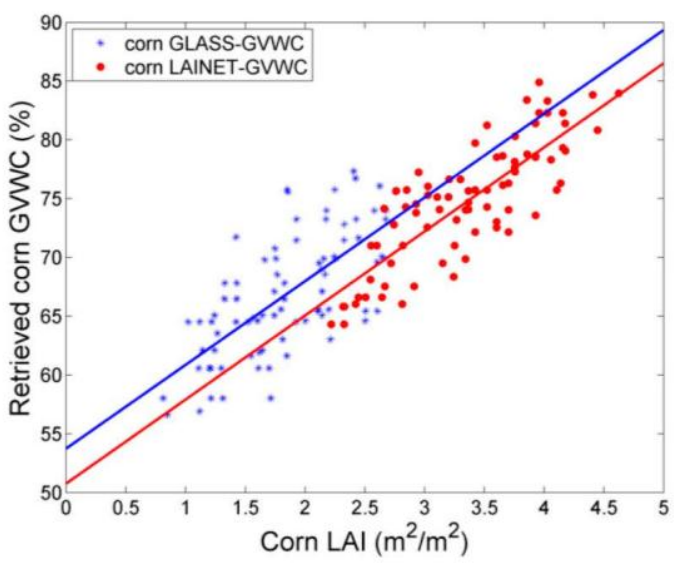

$\mathrm{b}$

Figure 11. (a) The comparison between the GLASS LAI product and the LAINet observation data; (b) The relationship between the corn LAI and the retrieved corn GVWC.

To explain this phenomenon further, we analyzed the inversion formula of the corn GVWC. According to the transformation of Equation (9), the corn GVWC was a function of the corn optical depth at L-band, the LAI, and the height and areal density of the corn stalks (Equation (14)).

$$
w=\frac{B-\tau}{C-A \cdot L A I}+D
$$

where $w$ is the corn GVWC (unit: \%), LAI is the corn leaf area index, $\tau$ is the corn optical depth, $A=a^{\prime}=0.1091, B=d^{\prime}-\left(b^{\prime} \cdot c^{\prime} / a^{\prime}\right), C=-c^{\prime}$, and $D=-b^{\prime} / a^{\prime}=0.24748$. The parameters $a^{\prime}, b^{\prime}$, $c^{\prime}$, and $d^{\prime}$ are coefficients; for more detailed information, please refer to Equations (9)-(11) in Section 2.2. 
Through an analysis of the data used in the corn GVWC retrieval, the numerator and the denominator of the first term on the right side of Equation (14) are shown to always exceed 0 during the corn seedling to the mature stage. The coefficients $A, B, C$, and $D$ always exceed 0 . Therefore, when the corn optical depth and the height and areal density of the corn stalks are fixed, the GVWC, i.e., the variable $w$ in Equation (14), is positively correlated with the $L A I$. This result indicates that a higher input $L A I$ is associated with a higher $w$, whereas a lower input $L A I$ is associated with a lower $w$. Figure $11 \mathrm{~b}$ shows that both the GLASS LAI and LAINET LAI display positive correlations with the corresponding retrieved corn GVWC, agreeing with other relevant studies [43,44]. The analysis above demonstrates that lower GLASS LAI data cause the corn GLASS-GVWC to be lower than the corn LAINET-GVWC at the identical time and place in the validation region. In other words, with more accurate LAI input data, more accurate results can be obtained, indicating that the proposed GVWC retrieval method was feasible and tractable when using accurate input data.

The assumptions in the GVWC retrieval algorithm may also produce errors, e.g., the assumption of $\omega$ (single scattering albedo) to be 0 (Section 2.1). Here, we analyzed and discussed this assumption based on the simulations (Section 2.2). The formula of $\omega$ is shown as Equation (15).

$$
\omega=\frac{Q s}{Q a+Q s}=\frac{Q s}{Q e}
$$

where $\omega$ is the single scattering albedo, $Q s$ and $Q a$ refer to the scattering and absorption cross sections, respectively, and $Q e=Q a+Q s$ refer to the attenuation cross section.

The simulations show that $Q s$ is very small and negligible, whereas $Q e$ is relatively large compared to $Q s$, indicating that the absorption cross section, $Q a$, is large (Figure 12). In addition, the radiation at L-band has a long wavelength that can penetrate low vegetation such as corn. In observations, the absorption effect of the microwave radiation signal is greater than the scattering effect at L-band when the viewing zenith angle is small. According to Equation 15, when the $Q s$ approximates 0 and the $Q e$ is greater than $0, \omega$ can be assume to be 0 . Previous studies $[20,45]$ showed that $\omega$ (generally between 0.04 and 0.12 at the C-band) displayed a low effect on the range of emissions from vegetated surfaces at microwave wavelengths. Whereas at L-band, Wigneron et al. [46] argued that for most low vegetation types, the $\omega$ is lower than 0.05 . Therefore, we assumed $\omega$ to be zero at L-band in this study similar to the assumptions in several other studies [20,21].

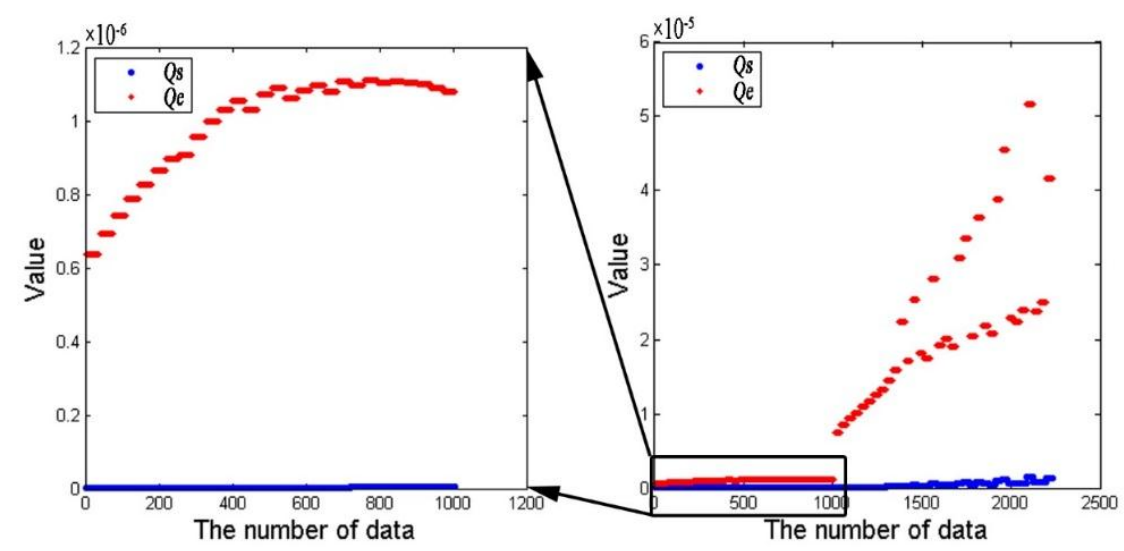

Figure 12. Comparison between the scattering cross section $Q s$ and the absorption cross section $Q e$ of the corn dielectric scatterers. 
In addition, the retrieval accuracy of the corn GVWC depends on the observation error and the algorithm error. The observation error is related to instabilities in the observations, such as mixed pixels and calibration errors. Although a quality control was performed for the PLMR TB, errors remain because the quality flags cannot ensure absolute correctness. The algorithm error mainly stems from the process of the algorithm development. Although the corn optical depth simulated by the model agrees with that calculations of Equation (9) and the two are uniformly distributed along the 1:1 line (Figure 3), errors may remain $\left(\mathrm{R}^{2}=0.9947\right.$ and $\left.\mathrm{RMSE}=0.0208\right)$.

\section{Conclusions}

Corn is one of the most widely distributed crops in the world, and its production plays an important role in national economies. Because the GVWC is highly related to the vegetation growing status, this variable appears to be superior to the VWC in evaluating the growth of and monitoring droughts for crops. In this study, a corn GVWC retrieval method based on microwave remote sensing data is developed and validated. The results show that accuracy of the retrieval algorithm is highly dependent on the accuracy of the input LAI data. This method will display an acceptable accuracy with accurate input LAI data. The Soil Moisture and Ocean Salinity (SMOS) satellite [47] provides a substantial number of L-band multi-angle TB measurements at a satellite scale, enabling the use of the algorithm proposed in this study to estimate the corn GVWC at regional scales. However, future additional efforts are required.

First, in this study, the work was only conducted in pixels with corn coverage higher than $60 \%$, indicating that the pixel heterogeneity was not included in our considerations. Moreover, because the study region is flat, the effects of the terrain were also not included. Li et al. [48] demonstrated that the topography does display influences on passive microwave remote-sensing signals over mountains. Therefore, the algorithm proposed in this study is not applicable for highly mixed pixels or for non-flat terrain. The difficulty in obtaining input parameters, i.e., the height and areal density of corn, may also limit the application of the algorithm. However, employing Lidar to obtain the height and areal density of the corn stalks may be practical at a regional scale. In addition, because the areal density of the corn stalks is approximately constant during the entire growing season within a certain region, this parameter will be easy to acquire. Additionally, the height of the corn stalks can also be obtained by the corn growing curve shown in Equations (12) and (13) with the re-regressed coefficients.

This method can be potentially applied to other types of crops and grasses by regressing a new set of coefficients in Equation (9) as long as the single scattering albedo can be assumed to be zero. This process can be achieved by modifying the configuration of vegetation parameters in Table 2 . Because of the coarse resolution of passive microwave remote sensing observations, this method cannot satisfy the requirements of precision agriculture. However, the observation ability of independent of weather of passive microwave remote sensing causes this method to be a beneficial supplement to optical remote sensing when monitoring the GVWC of crops. 


\section{Acknowledgments}

This study was jointly supported by National Basic Research Program of China (2013CB733406), National Natural Science Foundation of China (41171259 and 91125004). The authors are grateful to Xiyu Chen and Zhizhong Chen from Beijing Normal University for their valuable assistance

\section{Author Contributions}

Linna Chai proposed the idea, supervised the research work and finished the manuscript. Qi Wang conducted the research work including building and analyzing the simulated database, establishing the quantitative correlation, validating the retrieval results. All authors read and approved the manuscript.

\section{Conflicts of Interest}

The authors declare no conflict of interest.

\section{References}

1. Huang, J.; Chen, D.; Cosh, M.H. Sub-pixel reflectance unmixing in estimating vegetation water content and dry biomass of corn and soybeans cropland using normalized difference water index (NDWI) from satellites. Int. J. Remote Sens. 2009, 30, 2075-2104.

2. Zhao, T.; Zhang, L.; Bindlish, R.; Shi, J.; Jiang, L.; Li, Y.; Zhao, S.; Zhang, T.; Li, X. Estimating vegetation water content during a growing season of cotton. In Proceedings of the 2011 IEEE International Geoscience and Remote Sensing Symposium, Vancouver, BC, Canada, 24-29 July 2011; pp. 791-794.

3. Jackson, T.J.; Schmugge, T.J.; Wang J.R. Passive microwave sensing of soil moisture under vegetation canopies. Water Resour. Res. 1982, 18, 1137-1142.

4. Jackson, T.J.; Chen, D.; Cosh, M.; Li, F.; Anderson, M.; Walthall, C.; Doriaswamy, P.; Hunt, R.E. Vegetation water content mapping using Landsat data derived normalized difference water index for corn and soybeans. Remote Sens. Environ. 2004, 92, 475-482.

5. Ceccato, P.; Flasse, S.; Tarantola, S.; Jacquemoud, S.; Grégoire, J. Detecting vegetation leaf water content using reflectance in the optical domain. Remote Sens. Environ. 2001, 77, 22-33.

6. Grant, J.P.; Wigneron, J.P.; Drusch, M.; Williams, M.; Law, B.E.; Novello, N.; Kerr, Y. Investigating temporal variations in vegetation water content derived from SMOS optical depth. In Proceedings of the IEEE International Geoscience and Remote Sensing Symposium, Munich, Germany, 22-27 July 2012; pp. 3331-3334.

7. Jackson, T.J.; Schmugge, T.J. Vegetation effects on the microwave emission of soils. Remote Sens. Environ. 1991, 36, 203-212.

8. Le Vine, D.M.; Karam, M.A. Dependence of attenuation in a vegetation canopy on frequency and plant water content. IEEE Trans. Geosci. Remote Sens. 1996, 34, 1090-1096.

9. Jackson, T.J.; le Vine, D.M.; Hsu, A.Y.; Oldak, A.; Starks, P.J.; Swift, C.T.; Isham, J.D.; Haken M. Soil moisture mapping at regional scales using microwave radiometry: The Southern Great Plains hydrology experiment. IEEE Trans. Geosci. Remote Sens. 1999, 37, 2136-2151. 
10. Njoku, E.G.; Jackson, T.J.; Lakshmi, V.; Chan, T.K.; Nghiem, S.V. Soil moisture retrieval from AMSR-E. IEEE Trans. Geosci. Remote Sensi. 2003, 41, 215-229.

11. Van de Griend, A.A.; Wigneron, J.P. The B-factor as a function of frequency and canopy type at H-polarization. IEEE Trans. Geosci. Remote Sens. 2004, 42, 786-794.

12. Cheng, T.; Rivard, B.; Sánchez-Azofeifa, A.G.; Féret, J.B.; Jacquemoud, S.; Ustin, S.L. Predicting leaf gravimetric water content from foliar reflectance across a range of plant species using continuous wavelet analysis. J. Plant Physiol. 2012, 169, 1134-1142.

13. Viegas, D.X.; Viegas, M.T.S.P.; Ferreira, A.D. Moisture content of fine forest fuels and fire occurrence in central Portugal. Int. J. Wildl. Fire. 1992, 2, 69-86.

14. Ulaby, F.T.; Moore, R.K.; Fung, A.K. Microwave Remote Sensing: Active and Passive; Artech House Publishers: Norwood, MA, USA, 1986.

15. Mo, T.; Choudhury, B.J.; Schmugge, T.J.; Wang, J.R.; Jackson, T.J. A model for microwave emission from vegetation-covered fields. J. Geophys. Res. Oceans 1982, 87, 11229-11237.

16. Zhao, T.; Zhang, L.; Jiang, L.; Zhao, S.; Chai, L.; Jin, R. A new soil freeze/thaw discriminant algorithm using AMSR-E passive microwave imagery. Hydrol. Process. 2011, 25, 1704-1716.

17. Shi, J.; Jackson, T.J.; Tao, J.; Du, J.; Bindlish, R.; Lu, L.; Chen, K.S. Microwave vegetation indices for short vegetation covers from satellite passive microwave sensor AMSR-E. Remote Sens. Environ. 2008, 112, 4285-4300.

18. Owe, M.; Jeu, R.D.; Walker, J. A methodology for surface soil moisture and vegetation optical depth retrieval using the microwave polarization difference index. IEEE Trans. Geosci. Remote Sens. 2001, 39, 1643-1654.

19. Paloscia, S.; Macelloni, G.; Santi. E. Soil moisture estimates from AMSR-E brightness temperatures by using a dual-frequency algorithm. IEEE Trans. Geosci. Remote Sens. 2006, 44, 3135-3144.

20. Van de Griend, A.A.; Owe, M. Microwave vegetation optical depth and inverse modelling of soil emissivity using Nimbus/SMMR satellite observations. Meteorol. Atmos. Phys. 1994, 54, 225-239.

21. Wang, Q.; Chai, L. Estimating vegetation optical depth using L-band passive microwave airborne data in HiWATER. In Proceedings of the SPIE Asia Pacific Remote Sensing International Society for Optics and Photonics, San Diego, CA, USA, 8 November 2014.

22. Chen, K.; Wu, T.; Tsang, L.; Qin, L.; Shi, J.; Fung, A.K. Emission of rough surfaces calculated by the integral equation method with comparison to three-dimensional moment method simulations. IEEE Trans. Geosci. Remote Sens. 2003, 41, 90-101.

23. Le Vine, D.M.; Meneghini, R.; Lang, R.H. Scattering from arbitrarily orientated dielectric disks in the physical optics regime. J. Opt. Soc. Am. 1983, 73, 1255-1262.

24. Zhang, Z.; Zhang, L.; Xu, Y.; Liu, J.; Sun, G. Scattering and attenuation characteristics of corn at multiple frequencies and view angles by model simulation and truck-mounted microwave radiometer. J. Remote Sens. 2010, 14, 396-408.

25. Karam, M.A.; Fung, A.K. Electromagnetic scattering from a layer of finite length, randomly oriented, dielectric, circular cylinders over a rough interface with application to vegetation. Remote Sens. 1988, 9, 1109-1134.

26. Mätzler, C. Microwave (1-100 GHz) dielectric model of leaves. IEEE Trans. Geosci. Remote Sens. 1994, 32, 947-949. 
27. Karam, M.A.; Fung, A.K. Scattering from randomly oriented circular discs with application to vegetation. Radio Sci. 1983, 18, 557-565.

28. Karam, M.A.; Fung, A.K.; Antar, Y.M.M. Electromagnetic wave scattering from some vegetation samples. IEEE Trans. Geosci. Remote Sens. 1988, 26, 799-808.

29. Li, X.; Cheng, G.D.; Liu, S.M.; Xiao, Q; Ma, M.G.; Jin, R.; Che, T.; Liu, Q.H.; Wang, W.Z.; Qi, Y.; et al. Heihe Watershed Allied Telemetry Experimental Research (HiWATER): Scientific objectives and experimental design. Bull. Am. Meteorol. Soc. 2013, 94, 1145-1160.

30. Cheng, G.; Li, X.; Zhao, W.; Xu, Z.; Feng, Q.; Xiao, S.; Xiao, H. Integrated study of the water-ecosystem-economy in the Heihe River Basin. Natl. Sci. Rev. 2014, 13, 413-428.

31. Zhong, B.; Ma, P.; Nie, A.; Yang, A.; Yao, Y.; Lu, W.; Zhang, H.; Liu, Q. Land cover mapping using time series HJ-1/CCD data. Sci. China Earth Sci. 2014, 44, 967-977.

32. Hasan, S.; Montzka, C.; Rüdiger, C.; Ali, M.; Bogena, H.; Vereecken, H. Soil moisture retrieval from airborne L-band passive microwave using high resolution multispectral data. ISPRS J. Photogramm. Remote Sens. 2014, 91, 59-71.

33. Kang, J.; Jin, R.; Li, X. Regression kriging-based upscaling of soil moisture measurements from a wireless sensor network and multiresource remote sensing information over heterogeneous cropland. IEEE Geosci. Remote Sens. Lett. 2015, 12, 92-96.

34. Zhu, Z.; Tan, L.; Gao, S.; Jiao, Q. Observation on soil moisture of irrigation cropland by cosmic-ray probe. IEEE Geosci. Remote Sensi. Lett. 2015, 12, 472-476.

35. Che, T.; Li, X.; Gao, Y.; Jeff, W. HiWATER: Dataset of Airborne Microwave Radiometers (L Bands) Mission in the Middle Reaches of the Heihe River Basin on June 30, July4, July7, July10, July26, August1, and August 2, 2012; Heihe Plan Science Data Center: Lanzhou, China, 2013.

36. Zhao, X.; Liang, S.; Liu, S.; Yuan, W.; Xiao, Z.; Liu, Q.; Cheng, J.; Zhang, X.; Tang, H.; Zhang, X.; et al. The Global Land Surface Satellite (GLASS) remote sensing data processing system and products. Remote Sens. 2013, 5, 2436-2450.

37. Xiao, Z.; Liang, S.; Wang, J.; Chen, P.; Yin, X.; Zhang, L.; Song, J. Use of general regression neural networks for generating the GLASS leaf area index product from time-series MODIS surface reflectance. IEEE Trans. Geosci. Remote Sens. 2013, 52, 209-223.

38. Jin, R.; Li, X.; Yan, B.P.; Li, X.H.; Luo, W.M.; Ma, M.G.; Guo, J.W.; Kang, J.; Zhu, Z.L.; Zhao, S.J. A nested eco-hydrological wireless sensor network for capturing the surface heterogeneity in the midstream area of the Heihe River Basin, China. IEEE Geosci. Remote Sens. Lett. 2014, 11, 2015-2019.

39. Wang, W.; Huang, Y.; Xu, F.; Ma, C.; Wang, J. HiWATER: Dataset of Biomass Observed in the Middle Reaches of the Heihe River Basin; Heihe Plan Science Data Center: Lanzhou, China, 2013.

40. Qu, Y.; Wang, J.; Dong, J.; Jiang, F. Design and experiment of crop structural parameters automatic measurement system. Trans. Chin. Soc. Agri. Eng. 2012, 28, 160-165.

41. Qu, Y.; Zhu, Y; Han, W. HiWATER: Dataset of LAINet Observations in the Middle Reaches of the Heihe River Basin; Heihe Plan Science Data Center: Lanzhou, China, 2013.

42. Hanway, J.J. Growth stages of corn (Zea mays, L.). Agron. J. 1963, 55, 487-492. 
43. Baret, F.; Weiss, M.; Lacaze, R.; Camacho, F.; Makhmara, H.; Pacholczyk, P.; Smets, B. GEOV1: LAI and FAPAR essential climate variables and FCOVER global time series capitalizing over existing products. Part1: Principles of development and production. Remote Sens. Environ. 2013, 137, 299-309.

44. Zakharova, E.; Calvet, J.-C.; Lafont, S.; Albergel, C.; Wigneron, J.-P.; Pardé, M.; Kerr, Y.; Zribi, M. Spatial and temporal variability of biophysical variables in southwestern France from airborne L-band radiometry. Hydrol. Earth Syst. Sci. 2012, 16, 1725-1743.

45. Mladenova, I.E.; Jackson, T.J.; Njoku, E.; Bindlish, R.; Chan, S.; Cosh, M.H.; Holmes, T.R.H.; de Jeu, R.A.M.; Jones, L.; Kimball, J.; et al. Remote monitoring of soil moisture using passive microwave-based techniques - Theoretical basis and overview of selected algorithms for AMSR-E. Remote Sens. Environ. 2014, 144, 197-213.

46. Wigneron, J.P.; Kerr, Y.; Waldteufel, P.; Saleh, K.; Escorihuela, M.J.; Richaume, P.; Ferrazzoli, P.; Rosnay, P.D.; Gurney, R.; Calvet, J.C.; et al. L-band Microwave Emission of the Biosphere (L-MEB) model: Description and calibration against experimental data sets over crop fields. Remote Sens. Environ. 2007, 107, 639-655.

47. Kerr, Y.H.; Waldteufel, P.; Wigneron, J.P.; Delwart, S.; Cabot, F.; Boutin, J.; Escorihuela, M.J.; Font, J.; Reul, N.; Gruhier, C.; et al. The SMOS mission: New tool for monitoring key elements of the global water cycle. Proc. IEEE 2010, 98, 666-687.

48. Li, X.; Zhang, L.; Weihermüller, L; Jiang, L; Vereecken, H. Measurement and simulation of topographic effects on passive microwave remote sensing over mountain areas: A case study from the Tibetan Plateau. IEEE Trans. Geosci. Remote Sens. 2014, 52, 1489-1501.

(C) 2014 by the authors; licensee MDPI, Basel, Switzerland. This article is an open access article distributed under the terms and conditions of the Creative Commons Attribution license (http://creativecommons.org/licenses/by/4.0/). 